\title{
Nonlocal constitutive equations of elasto-visco-plasticity coupled with damage and temperature
}

\author{
Weijie Liu ${ }^{1,2}$, Khemais Saanouni $^{2, a}$, Carl Labergère ${ }^{2}$, Houssem Badreddine ${ }^{2}$ Samuel Forest ${ }^{3}$ and Ping Hu${ }^{1}$ \\ ${ }^{1}$ Faculty of Vehicle Engineering and Mechanics, Dalian University of Technology, 2 Rue Linggong, 116024 Dalian, China \\ ${ }^{2}$ ICD/LASMIS, UMR-CNRS 6281, University of Technology of Troyes, 12 Rue Marie Curie BP2060, 10000 Troyes Cedex, France \\ ${ }^{3}$ Centre des matériaux, UMR-CNRS 7633, MINES ParisTech, BP 87, 91003 Evry Cedex, France
}

\begin{abstract}
In this paper, the nonlocal anisothermal elasto-visco-plastic constitutive equations strongly coupled with ductile isotropic damage, nonlinear isotropic hardening and kinematic hardening are developed to model the material behaviour under finite strain. The new micromorphic variable of damage is introduced into the principle of virtual power and new additional balance equations are obtained. Thermodynamically-consistent nonlocal constitutive equations are then deduced. The evolution equations are deduced from the generalized normality rule for the NortonHoff visco-plastic potential. This model is used to simulate various material responses under different velocities at high temperature. The micromorphic parameters of damage: micromorphic density and $\mathrm{H}$ moduli are studied to examine the effects of micromorphic damage. Biaxial tension is performed to make a comparison between the local damage model and the micromorphic damage model.
\end{abstract}

\section{Introduction}

Nowadays, economic and environmental demands are forcing the industrialists to design lightweight mechanical components. The large-scale complex metallic components are manufactured using hot or cold forming processes under relatively extreme conditions, such as complex multi-axial loading, high strain rate, and high temperature. Even for the experienced engineers, it is hardly possible to estimate the finite deformations.

The fully local Cauchy constitutive equations have been well established to model the induced material softening behaviour due to thermal, damage and other microstructure-dependent phenomena. However, the solutions of the fully local constitutive equations are highly sensitive to the space and time discretization. The natural way to overcome this drawback is to account for an appropriate neighbourhood effect of each material point by introducing some characteristic lengths, representative of the materials' microstructures, into constitutive equations ${ }^{[1]}$.

The generalized continuum mechanics makes possible the straightforward introduction of characteristic lengths into the constitutive equations of materials with microstructure. During the 1960s, motivated by the original works of the Cosserat ${ }^{[2]}$ brothers, many theoretical works have been devoted to the mechanics of generalized continua to solve various problems in mechanics of solids and fluids left without satisfactory solution in the framework of classical local continuum mechanics as found in Mindlin ${ }^{[3-5]}$; Eringen and Suhubi ${ }^{[6]}$; Eringen ${ }^{[7]}$.

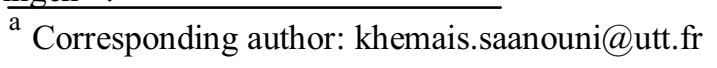

As summarised by Forest ${ }^{[8,9]}$, all these generalized continuum theories, which are still based on the assumption of local action (Truesdell and Noll ${ }^{[10]}$ ), can be classified into two classes: (i) the higher grade continua and (ii) the higher order continua. Higher grade continua are those based on higher order spatial derivatives of the displacement field. While, higher order continua are based on the introduction of additional degrees of freedom $^{[11]}$. A third class of generalized continuum theories is the so called strictly nonlocal continuum field theories, well summarized by Eringen ${ }^{[12]}$ where a unified foundation of the basic field equations are presented and are not based on the principle of the local action.

The micromorphic theory was proposed by (Eringen and Suhubi ${ }^{[6]}$; Mindlin $\left.{ }^{[3]}\right)$. It envisions a material body as a continuous collection of deformable particles; each possesses finite size and inner structure and requires supplying additional degrees of freedom (dof) to a material point ${ }^{[13]}$. On the other hand, classical continuum mechanics considers a material body as a continuous collection of material points, each with infinitesimal size and no inner structure and usually needs displacements as dofs. The purpose to go beyond the classical continuum mechanics is to take into account the microstructure of the material in question while still keeping the advantages of continuum theory. The micromorphic approach can be applied to any macroscopic quantity in order to introduce a character length scale in the original classical continuum model in a systematic way, as presented by (Fores $\left.{ }^{[9]}\right)$. From the comparison between nonlocal and micromorphic theories, Forest and Aifantis ${ }^{[14]}$ concluded 
that when the micromorphic variable remains as close as possible to the plastic strain, the micromorphic model reduces to the strain gradient theory.

The main goal of this paper is to develop and examine the generalized anisothermal elasto-visco-plastic constitutive equations strongly coupled with ductile damage, nonlinear isotropic and kinematic hardening. The effects on the thermal viscoplasticity from the micromorphic damage are examined from the parametrical study and biaxial tension test.

\section{Theoretical aspects}

In this section, a set of fully coupled constitutive equations will be derived from the state and dissipation potentials in the form of elasto-visco-plasticity and anisothermal formulations, coupled with the local isotropic and kinematic hardening, local isotropic damage, micromorphic isotropic and kinematic hardening, and micromorphic damage, under the framework of thermodynamic irreversible processes with state variables.

\subsection{The Extended principle of virtual power}

The generalized virtual power of internal forces is extended by the micromorphic damage, isotropic and kinematic hardenings, and their first gradients:

$$
P_{\text {int }}\left(\vec{u}^{*}, \vec{d}^{*}\right)=-\int_{V}\left(\underline{\sigma}: \dot{\nabla}_{\vec{u}}^{*}+\dot{\bar{y}}^{*}+\overrightarrow{\bar{Y}} \cdot \nabla \dot{\vec{d}}^{*}\right) d V
$$

where, $\underline{\sigma}$ is the Cauchy stress tensor. $\breve{Y}$ and $\vec{Y}$ are the stress-like variables with respect to micromorphic damage $\breve{d}$ and its first gradient respectively.

The extended virtual power of external forces can be written in the additive form: classical local contributions and additional micromorphic contributions:

$$
\begin{aligned}
P_{\text {ext }} & =\rho \int_{V}\left(\vec{f}^{u} \cdot \dot{\vec{u}}^{*}\right) d V+\int_{\Gamma}\left(\vec{F}^{u} \cdot \dot{\vec{u}}^{*}\right) d S \\
& +\rho \int_{V}\left(f^{\breve{d}} \dot{\vec{d}}^{*}+\vec{f}^{g \breve{d}} \cdot \vec{\nabla} \dot{\vec{d}}^{*}\right) d V+\int_{\Gamma}\left(F^{\breve{d}} \dot{\vec{d}}^{*}\right) d S
\end{aligned}
$$

where, the small $f$ indicates the generalized body force associated with the displacement, micromorphic degree of freedoms and their first gradients. The capital $F$ is the generalized contact surface forces of displacement and micromorphic dofs respectively.

In the same way, the generalized virtual power of inertia forces is given below:

$$
P_{a}=\rho \int_{V}\left(\ddot{\vec{u}} \cdot \dot{\vec{u}}^{*}+\zeta_{\vec{d}} \ddot{\bar{d}}^{*}\right) d V
$$

where, $\zeta_{\bar{d}}$ is a scale factor which maps the local density to the micromorphic level ${ }^{[15]}$.

Applying the generalized virtual power for any given kinematical admissible fields, we may obtain the classical equilibrium equation and three additional balance equations:

$$
\begin{cases}\vec{\nabla} \cdot \underline{\sigma}+\rho \vec{f}^{u}=\rho \ddot{\vec{u}} & \text { in } \Omega \\ \underline{\sigma} \cdot \vec{n}=\vec{F}^{u} & \text { on } \Gamma\end{cases}
$$

$$
\left\{\begin{array}{cc}
(\vec{\nabla} \cdot \overrightarrow{\bar{Y}}+\breve{Y})+\rho\left(f^{\breve{d}}-\dot{\nabla} \cdot \dot{f}^{g \breve{d}}\right)=\rho \zeta_{\bar{D}} \ddot{\vec{d}} & \text { in } \Omega \\
\left(\overrightarrow{\bar{Y}}-\rho \vec{f}^{g \breve{d}}\right) \cdot \vec{n}=F^{\bar{d}} & \text { on } \Gamma
\end{array}\right.
$$

\subsection{Thermodynamic irreversible processes}

We assume that the energy conservation holds for the micromorphic continuum of the first order. Using the kinetic energy theorem, the local form of the rate of specific internal energy ( $1^{\text {st }}$ principle of thermodynamics $)$ is enhanced with the additional micromorphic dofs:

$$
\rho \dot{e}=\underline{\sigma}: \underline{\dot{\varepsilon}}+(\rho \varsigma-\vec{\nabla} \cdot \vec{q})+(\dot{Y} \dot{\vec{d}}+\overrightarrow{\vec{Y}} \cdot \vec{\nabla} \dot{\vec{d}})
$$

where, $\varsigma$ is the volume heat generation. $\vec{q}$ is the heat flux vector across the boundary surface.

According to the well-known Clausius-Duhem inequality and the relations between the specific internal energy and specific Helmholtz free energy, we may have the following inequality:

$$
\underline{\sigma}: \underline{\dot{\varepsilon}}-\rho(s \dot{T}+\dot{\psi})-\frac{\vec{q}}{T} \cdot \vec{\nabla} T+(\vec{Y} \dot{\vec{d}}+\overrightarrow{\vec{Y}} \cdot \vec{\nabla} \dot{\vec{d}}) \geq 0
$$

where, $s$ is the specific entropy. $T$ represents the temperature.

As in local continuum mechanics, the state potential is assumed to be a closed concave function of temperature and convex function of all the classical local state variables elastic strain, isotropic and kinematic hardening, and local damage as well as the micromorphic damage, micromorphic isotropic and kinematic hardening, and their first gradients. With the assumption of additive decomposition of total strain rate, the Clausius-Duhem inequality Eq.(7) can be expressed as:

$$
\begin{aligned}
& \left(\underline{\sigma}-\rho \frac{\partial \psi}{\partial \underline{\varepsilon}^{e}}\right): \underline{\dot{\varepsilon}}^{e}+\rho\left(s+\frac{\partial \psi}{\partial T}\right) \dot{T}+\left(\vec{Y}-\rho \frac{\partial \psi}{\partial \vec{d}}\right) \dot{\bar{d}} \\
& +\left(\overrightarrow{\bar{Y}}-\rho \frac{\partial \psi}{\partial \vec{\nabla} \tilde{d}}\right) \cdot \vec{\nabla} \dot{\vec{d}}+\underline{\sigma}: \underline{\dot{\varepsilon}}^{v p} \\
& -\left(\rho \frac{\partial \psi}{\partial d} \dot{d}+\rho \frac{\partial \psi}{\partial r} \dot{r}+\rho \frac{\partial \psi}{\partial \underline{\alpha}}: \underline{\dot{\alpha}}\right)-\frac{\vec{q}}{T} \cdot \vec{\nabla} T \geq 0
\end{aligned}
$$

Generally, if we assume that overall the formulas $\left(\underline{\sigma}-\rho \frac{\partial \psi}{\partial \underline{\varepsilon}^{e}}\right),\left(s+\frac{\partial \psi}{\partial T}\right),\left(\bar{Y}-\rho \frac{\partial \psi}{\partial \vec{d}}\right)$ and $\left(\overrightarrow{\vec{Y}}-\rho \frac{\partial \psi}{\partial \vec{\nabla} \vec{d}}\right)$ do not depend on their rates respectively and the micromorphic variables do not dissipate, the following state relations and pure local residual dissipation are obtained:

- The classical local state relations:

$$
\begin{array}{ll}
\underline{\sigma}=\rho \frac{\partial \psi}{\partial \underline{\varepsilon}^{e}} & s=-\frac{\partial \psi}{\partial T} \\
\underline{X}=\rho \frac{\partial \psi}{\partial \underline{\alpha}} & R=\rho \frac{\partial \psi}{\partial r} \quad Y=-\rho \frac{\partial \psi}{\partial d}
\end{array}
$$

- The micromorphic (nonlocal) state relations:

$$
\breve{Y}=\rho \frac{\partial \psi}{\partial \breve{d}} \quad \overrightarrow{\bar{Y}}=\rho \frac{\partial \psi}{\partial \vec{\nabla} \breve{d}}
$$


- The residual dissipation:

$$
\phi_{i n}=\underline{\sigma}: \underline{\dot{\varepsilon}}^{v p}-\underline{X}: \underline{\dot{\alpha}}-R \dot{r}+Y \dot{d}-\frac{\vec{q}}{T} \cdot \vec{\nabla} T \geq 0
$$

\subsection{The heat equation}

Using the local form of $1^{\text {st }}$ principle of thermodynamic Eq.(6), the state relations Eqs.(9)-(10) and the relations of internal energy and Helmholtz free energy, we may have the following enhanced heat equation:

$$
\underline{\sigma}: \underline{\dot{\varepsilon}}^{v p}-\underline{X}: \underline{\dot{\alpha}}-R \dot{r}+Y \dot{d}+(\rho \varsigma+\vec{\nabla} \cdot \vec{q})-\rho \dot{s} T=0
$$

Substituting the state relation of entropy into the above equation:

$$
\begin{aligned}
& \underline{\sigma}: \underline{\dot{\varepsilon}}^{I R}+Y \dot{d}-R \dot{r}-\underline{X}: \underline{\dot{\alpha}}+(\rho \varsigma-\vec{\nabla} \cdot \vec{q})-\rho T \frac{\partial(s)}{\partial T} \dot{T} \\
& +T\left(\begin{array}{l}
\frac{\partial \underline{\sigma}}{\partial T}: \underline{\dot{\varepsilon}}^{e}-\frac{\partial Y}{\partial T} \dot{d}+\frac{\partial R}{\partial T} \dot{r}+\frac{\partial \underline{X}}{\partial T}: \underline{\dot{\alpha}} \\
+\frac{\partial \breve{Y}}{\partial T} \dot{d}+\frac{\partial Y}{\partial T} \cdot \dot{\nabla} \dot{d}
\end{array}\right)=0
\end{aligned}
$$

If we assign:

$$
R_{p l}=\left(\begin{array}{l}
\frac{\partial \underline{\sigma}}{\partial T}: \dot{\underline{\varepsilon}}^{e}-\frac{\partial Y}{\partial T} \dot{d}+\frac{\partial R}{\partial T} \dot{r}+\frac{\partial \underline{X}}{\partial T}: \underline{\dot{\alpha}} \\
+\frac{\partial \breve{Y}}{\partial T} \dot{\vec{d}}+\frac{\partial \dot{Y}}{\partial T} \cdot \dot{\nabla} \dot{\bar{d}}
\end{array}\right)
$$

The final heat equation can be rewritten as:

$$
\rho C_{v} \dot{T}-\rho \varsigma+\vec{\nabla} \cdot \vec{q}-\phi_{i n}-R_{p l} T=0
$$

where, $C_{v}$ is the specific heat capacity for constant volume.

Clearly, $R_{p l}$ comprises the additional contributions to the heat equation from the micromorphic variables.

\section{An example of micromorphic constitutive and evolution equations}

In this section, a specific state potential is constructed for the anisothermal isotropic elasto-visco-plasticity model fully coupled with isotropic local damage, isotropic and kinematic hardening and isotropic micromorphic damage. The damaged state variables are applied to the local state variables according to the assumption of the equivalence of total energy developed in references ${ }^{[1,11,16-18]}$.

\subsection{Micromorphic state potential and associated state relations}

For simplification, we also postulate that only the local damage is coupled with the micromorphic damage. With these assumptions, a quadratic the Helmholtz free energy can be expressed as:

$$
\begin{aligned}
\rho \psi & =\frac{1}{2}\left(\underline{\tilde{\varepsilon}}^{e}: \underline{\Lambda}: \underline{\tilde{\varepsilon}}^{e}+\frac{2}{3} C \underline{\tilde{\alpha}}: \underline{\tilde{\alpha}}+Q \tilde{r}^{2}\right) \\
& +\frac{1}{2} \breve{H}(d-\breve{d})^{2}+\frac{1}{2} \breve{H}^{g} \vec{\nabla} \breve{d} \cdot \vec{\nabla} \breve{d} \\
& -\rho C_{v} \frac{\left(T-T_{0}\right)^{2}}{2 T_{0}}-\left(T-T_{0}\right) P \underline{1}: \underline{\tilde{\varepsilon}}^{e}
\end{aligned}
$$

where, $\underline{\Lambda}=\lambda_{e} \underline{1} \otimes \underline{1}+2 \mu_{e} \underline{\underline{1}}$ is the fourth order elastic tensor expressed with Lamé's parameters. $C$ and $Q$ are the macro moduli of local kinematic and isotropic hardening respectively. $\breve{H}$ and $\breve{H}^{g}$ are the moduli of coupled local and micromorphic damage and its first gradient respectively. $P$ is a scalar modulus for isotropic thermal expansion.

Using the state relations Eqs.(9)-(10), we may have the following expressed local stress-like variables:

$$
\begin{aligned}
\underline{\sigma} & =\underline{\tilde{\Lambda}}: \underline{\varepsilon}^{e}-\left(T-T_{0}\right) \tilde{P} \underline{1} \\
s & =\frac{C_{v}}{T_{0}}\left(T-T_{0}\right)+\frac{1}{\rho} \tilde{P} \underline{1}: \underline{\varepsilon}^{e} \\
\underline{X} & =\frac{2}{3} \tilde{C} \underline{\alpha} \quad R=\tilde{Q} r \\
Y & =\frac{1}{2}\left(\underline{\varepsilon}^{e}: \underline{\Lambda}: \underline{\varepsilon}^{e}+\frac{2}{3} C \underline{\alpha}: \underline{\alpha}+Q r^{2}\right) \\
& -\breve{H}(d-\breve{d})-\frac{1}{2 \sqrt{1-d}}\left(T-T_{0}\right) P \underline{1}: \underline{\varepsilon}^{e}
\end{aligned}
$$

And nonlocal stress-like variables:

$$
\breve{Y}=-\breve{H}(d-\breve{d}) \quad \overrightarrow{\bar{Y}}=\breve{H}^{g} \vec{\nabla} \breve{d}
$$

It's clear that if the micromorphic effects are neglected, $\breve{H}=\breve{H}^{g}=0$, then all the micromorphic state variables given by Eq.(18) vanish leading to zero contribution of the nonlocal terms. In that case the classical fully coupled local state relations originally given in Saanouni et $\mathrm{a}^{[1,16]}$. are recovered.

\subsection{Dissipation analysis and evolution equations}

For the case of the single potential, fully isotropic damageable thermo-elasto-visco-plasticity with ductile damage, we postulate that the overall viscoplastic potential $\varphi^{*}(\underline{\tilde{\sigma}}, \underline{\tilde{X}}, \tilde{R} ; T)$ is additively decomposed ${ }^{[1]}$ into two contributions representing respectively the NortonHoff viscoplastic flow $\varphi_{v p}^{*}(\underline{\tilde{\sigma}}, \underline{\tilde{X}}, \tilde{R} ; T)$ and the damage potential $\varphi_{d}^{*}(d ; T)$ :

$$
\begin{aligned}
\varphi_{v p}^{*} & =\frac{K^{v}(T)}{m_{v}(T)+1}\left\langle\frac{F_{p}(\underline{\tilde{\sigma}}, \underline{\tilde{X}}, \tilde{R} ; T)}{K^{v}(T)}\right\rangle^{m_{v}(T)+1} \\
\varphi_{d}^{*} & =\frac{S_{d}(T)}{\left(s_{d}(T)+1\right)(1-d)^{\beta_{d}(T)}}\left\langle\frac{Y-Y_{0}(T)}{S_{d}(T)}\right\rangle^{\left(s_{d}(T)+1\right)} \dot{p}
\end{aligned}
$$

where, $K^{v}$ and $m_{v}$ are two constants which are characteristic of material viscosity and $F_{p}(\underline{\tilde{\sigma}}, \underline{\tilde{X}}, \tilde{R} ; T)$ is expressed in term of fully isotropic case: 
$F_{p}=f_{p}+\frac{3}{4} \frac{a \underline{X}: \underline{X}}{C(1-d)}+\frac{1}{2} \frac{b R^{2}}{Q(1-d)}-\frac{1}{3} a C \underline{\tilde{\alpha}}: \underline{\tilde{\alpha}}-\frac{1}{2} b Q \tilde{r}^{2}$

with $f_{p}=\|\underline{\tilde{q}}-\underline{\tilde{X}}\|-\tilde{R}-\sigma_{y}$ is the classical yield function. $\dot{p}$ is the accumulated viscoplastic strain rate.

The evolution equations deduced from these potentials, using the generalized normality rule are:

- Viscoplastic strain rate:

$$
\underline{D}^{v p}=\left\langle\frac{f_{p}}{K^{v}}\right\rangle^{m_{v}} \frac{\partial f_{p}}{\partial \underline{\sigma}}=\dot{\lambda}_{v p} \underline{\tilde{n}}_{f}
$$

where, $\underline{\tilde{n}}_{f}=\frac{\partial f_{p}}{\partial \underline{\sigma}}=\frac{3}{2} \frac{1}{\sqrt{1-d}} \frac{\left(\underline{\sigma}^{d e v}-\underline{X}\right)}{\left\|\underline{\sigma}^{d e v}-\underline{X}\right\|}$ and the multiplier $\dot{\lambda}_{v p}$, depending on the form of the potential, is given as follow:

$$
\dot{\lambda}_{v p}=\left\langle\frac{f_{p}}{K^{v}}\right\rangle^{m_{v}}
$$

- Kinematic hardening strain rate:

$$
\dot{\alpha}=-\left\langle\frac{f_{p}}{K^{v}}\right\rangle^{m_{v}} \frac{\partial F_{p}}{\partial \underline{X}}=\dot{\lambda}_{v p}\left(\underline{\tilde{n}}_{f}-a \underline{\alpha}\right)
$$

- Isotropic hardening strain rate:

$$
\dot{r}=-\left\langle\frac{f_{p}}{K^{v}}\right\rangle^{m_{v}} \frac{\partial F_{p}}{\partial R}=\frac{\dot{\lambda}_{v p}}{\sqrt{1-d^{v}}}(1-b \tilde{r})
$$

- Isotropic ductile damage rate:

$$
\dot{d}=\frac{\dot{p}}{(1-d)^{\beta_{d}}}\left\langle\frac{Y-Y_{0}}{S_{d}}\right\rangle^{s_{d}}=\dot{p} \widehat{Y}
$$

From Eq.(22), we define the equation for the actual viscoplasticity yield surface in the form:

$$
f_{v p}=f_{p}-K^{v}\left(\dot{\lambda}_{v p}\right)^{\frac{1}{m_{v}}}=f_{p}-\sigma^{v}=0
$$

As expected, viscous stress is very dependent on the form of the viscoplastic potential and is defined as follow:

$$
\sigma^{v}=K^{v}\left(\dot{\lambda}_{v p}\right)^{\frac{1}{m_{v}}}
$$

\section{Numerical implementation}

In this section, we perform the parametrical study of the proposed model of anisothermal damaged isotropic elasto-visco-plasticity fully coupled isotropic, kinematic hardening and micromorphic isotropic damage. The material property under the reference temperature $\left(20^{\circ} \mathrm{C}\right)$ is given in the below table.

Table 1. Material parameters under reference temperature.

\begin{tabular}{|c|c|c|}
\hline Name & Symbol & Value \\
\hline Young's modulus & $E(T)$ & $205000 \mathrm{MPa}$ \\
\hline Poisson ratio & $v$ & 0.3 \\
\hline
\end{tabular}

\begin{tabular}{|c|c|c|}
\hline Yield stress & $\sigma_{y}(T)$ & $792 \mathrm{MPa}$ \\
\hline $\begin{array}{c}\text { Isotropic hardening } \\
\text { modulus }\end{array}$ & $Q(T)$ & $320 \mathrm{MPa}$ \\
\hline $\begin{array}{c}\text { Kinematic hardening } \\
\text { modulus }\end{array}$ & $C(T)$ & 0.6 \\
\hline & $a$ & $2000 \mathrm{MPa}$ \\
\hline Damage factors & $S_{d}(T)$ & 20 \\
\hline & $s_{d}$ & 2 \\
\hline & $\beta_{d}$ & 1 \\
\hline & $Y_{0}$ & 0 \\
\hline & $\gamma$ & 1.8 \\
\hline Visco-plasticity factors & $K^{v}$ & $793.8 \mathrm{MPa}$ \\
\hline & $m_{v}$ & 2.7 \\
\hline
\end{tabular}

As usual the material moduli are temperature dependent, the following relations are assumed:

$$
P(T)=P_{0}\left[1-G^{\alpha}(T)\right]
$$

where, $G(T)=\frac{T-T_{0}}{T_{f}-T_{0}} \geq 0$ and $T_{0}$ is the reference temperature. $T_{f}$ is the fusion temperature for the specific material. $\alpha$ is a coefficient. For Young's modulus $\alpha=4$; for capital $\mathrm{S}$ of damage factor $\alpha=1.08$ and for other modules it equals to 1.03 .

\subsection{Anisothermal viscoplasticity with local damage for a material point}

In this section, the parametrical studies of temperature, load velocity, micromorphic density and its moduli are performed in a material point to examine the response of the proposed anisothermal elasto-visco-plastic model coupled with local and micromorphic isotropic damage, local isotropic hardening and kinematic hardening, .

Figure 1 illustrates the thermal effects on the evolutions of equivalent stress based on the proposed constitutive equations coupled with local damage and local mixed hardening. The accumulated plastic strain (max: 0.652; min: 0.592) increases and the tensile stress (max: $1108.72 \mathrm{MPa}$; min: $416.91 \mathrm{MPa}$ ) decreases steadily as temperature increases.

Figure 2 shows the four types of evolutions of local damage under different strain rates (velocity) (from 1.0E$04 \mathrm{~s}^{-1}$ to $0.1 \mathrm{~s}^{-1}$ ) under the initial temperature $1000^{\circ} \mathrm{C}$. Clearly, the evolution of local damage is accelerated by the increasing strain rate. The fracture plastic strains are $0.652,0.648,0.636$ and 0.605 respectively. 


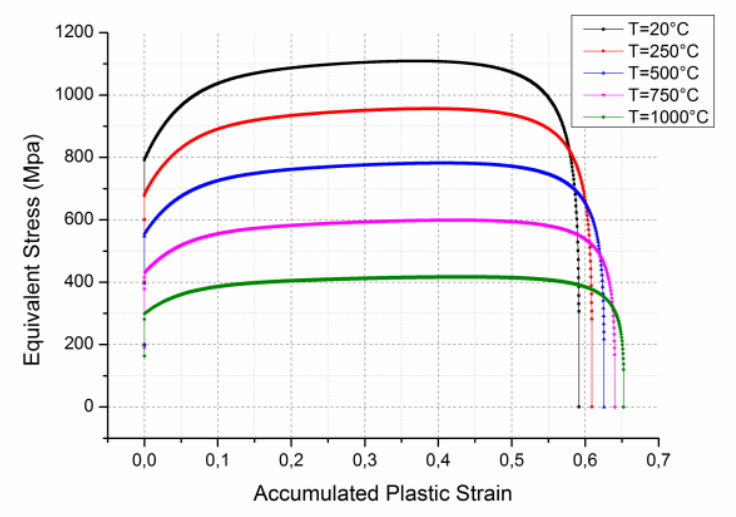

Figure 1. Stress-strain curves for different temperatures with the same strain rate $1.0 \mathrm{E}-04 \mathrm{~s}^{-1}$.

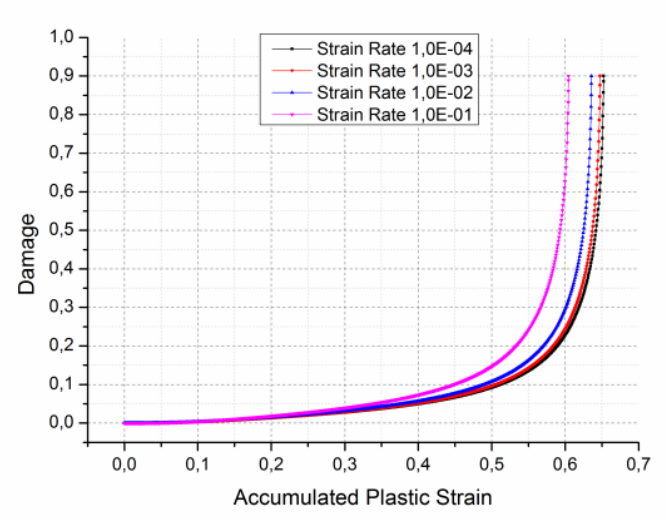

Figure 2. Evolution of damage for different loading velocity with the same temperature $1000^{\circ} \mathrm{C}$.

\subsection{Biaxial tension for local damage with FE}

The geometric size and boundary conditions of the sample are shown in Figure 3. For simplifications, three different initial temperatures $\left(20^{\circ} \mathrm{C}, 500^{\circ} \mathrm{C}\right.$ and $\left.1000^{\circ} \mathrm{C}\right)$, and four velocities $(2.0 \mathrm{E}-04 \mathrm{~mm} / \mathrm{s}, 2.0 \mathrm{E}-05 \mathrm{~mm} / \mathrm{s}, 2.0 \mathrm{E}-$ $06 \mathrm{~mm} / \mathrm{s}$ and $2.0 \mathrm{E}-07 \mathrm{~mm} / \mathrm{s}$ ) are performed on a single finite element in this example. The approximate strain rates are $1.0 \mathrm{E}-01 \mathrm{~s}^{-1}, 1.0 \mathrm{E}-02 \mathrm{~s}^{-1}, 1.0 \mathrm{E}-03 \mathrm{~s}^{-1}$ and $1.0 \mathrm{E}-04 \mathrm{~s}^{-1}$ respectively.

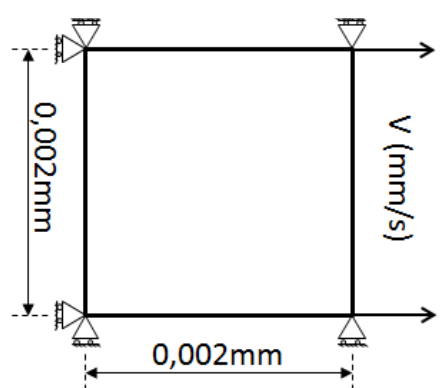

Figure 3. Scheme of tension test.

Figure 4 summarizes the material responses of the local damage model, in terms of equivalent stress accumulated plastic strain curves, for 7 cases including three temperatures and three velocities. From this figure the viscoeffects are very clear: when the temperature increases the ductile plasticity is extended and the final fracture plastic strain is also enlarged $\left(0.591\right.$ in $20^{\circ} \mathrm{C}$, 0.626 in $500^{\circ} \mathrm{C}$ and 0.653 in $1000^{\circ} \mathrm{C}$ with velocity $2.0 \mathrm{E}-$ $07 \mathrm{~mm} / \mathrm{s})$. However, both the initial yield stress (792.00MPa, 547.09MPa and 281.12MPa) and hardening effects are decreased. The opposite tendency of the fracture plastic strain is observed for the raise of loading velocity at each temperature, while the equivalent stress after yield point shows significant differences $(31.24 \mathrm{MPa}$ for $500^{\circ} \mathrm{C}$ and $66.85 \mathrm{MPa}$ for $1000^{\circ} \mathrm{C}$ ). In Figure 5, it illustrates the different evolutions of local damage for the same seven cases. It's clear that the evolution of damage is delayed by the raise of the temperature and the decelerated load velocity.

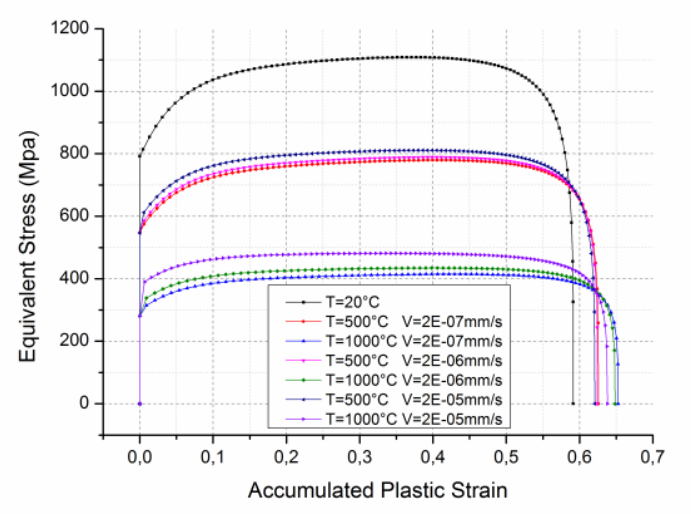

Figure 4. Stress-strain curves for different temperature and load velocity coupled with local damage.

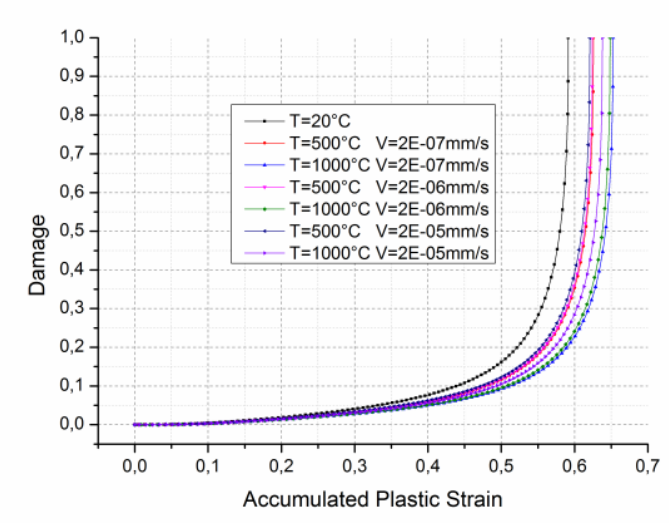

Figure 5. Evolution of local damage for different temperature and load velocity.

\subsection{Choices of micromorphic parameters with FE}

Let us consider the choices of micromorphic parameters including micromorphic density, the $\mathrm{H}$ moduli for the coupled local damage and micromorphic damage and the gradient of micromorphic damage. In this section, all the simulations are performed under temperature $500^{\circ} \mathrm{C}$ and load velocity $2.0 \mathrm{E}-05 \mathrm{~mm} / \mathrm{s}$.

In Figure 6, it illustrates the stress-strain curves and the evolutions of damage for different micromorphic density (0.1 100 times of local density) with given $\mathrm{H}$ modules equal to $1000 \mathrm{MPa}$. From the theoretical formula Eq.(5), we can see that the micromorphic density will be 
used to calculate the accelerate of micromorphic damage at each time step, which determines the incremental micromorphic damage and affects the local damage. In this figure, it clearly represents that the larger micromorphic density evidently slows the evolutions of local damage and enlarged the ductile plasticity of the material.

Figure 7 represents the effects of different choices of $\mathrm{H}$ modules $(1.0 \sim 1000 \mathrm{MPa})$ for the stress-strain curves and the evolution of local damage. From the state relations of stress-like variable with respect to local damage Eq.(17), we know that the evolution of the local damage is affected by the micromorphic damage through the $\mathrm{H}$ modules. In this figure, it describes that the larger $\mathrm{H}$ modules the slower evolution of local damage. We can also find this character from Eq.(17), the term of coupled local and micromorphic damage has a negative sign, which induces the reduction of the stress-like variable $\mathrm{Y}$ of local damage.

Compared with the curves for local damage, both of the figures imply that when the micromorphic density or the $\mathrm{H}$ modules approach to zero, the material response becomes closer and closer to the one from local damage.

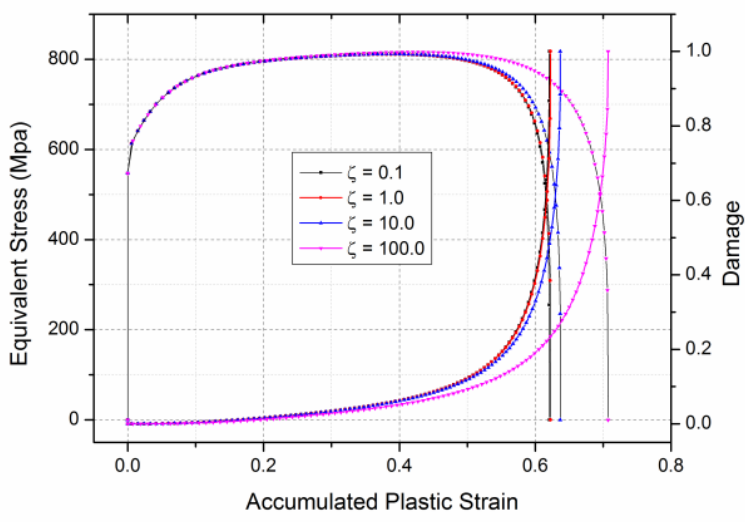

Figure 6. Stress-strain curves and evolution of damage for some values of micromorphic density.

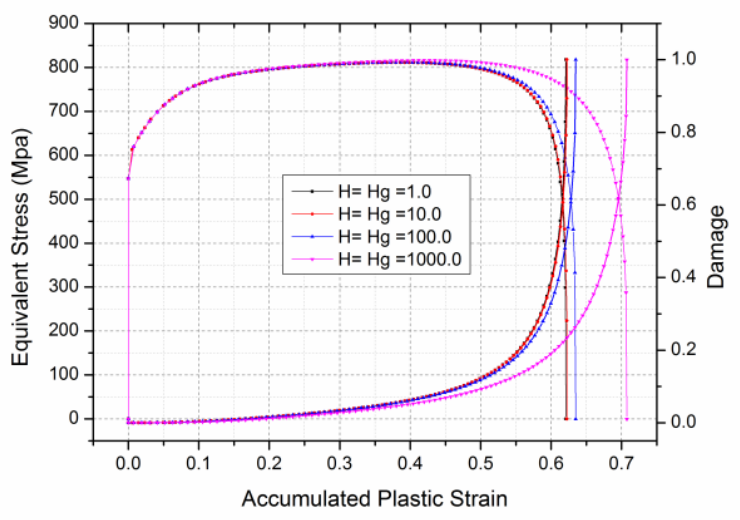

Figure 7. Stress-strain curves and evolution of damage for some values of micromorphic $\mathrm{H}$ modules.

\subsection{Biaxial tension for micromorphic damage with FE}

After the analysis of parameters, we have a conclusion that the smaller micromorphic density and $\mathrm{H}$ modules gives the more approximate result to the ones from the local damage formulas. In absence of any experimental indication, the micromorphic parameters: density 100 times, $\mathrm{H}$ modules $1000 \mathrm{MPa}$, will be considered to perform the biaxial tension test in this section.

In Figure 8 and Figure 9, it gives the stress-strain curves and the evolutions of local damage of 9 cases (three temperatures and four velocities). It's clear that, in this choice of parameters, the fracture plastic strain is significantly improved and the evolution of local damage is apparently delayed. Compared with the curves from local damage formulas Figure 4 and 5, it's apparently that the maximum tensile stresses have very little differences for the four velocities on each temperature. However, the final fracture accumulated plastic strain grows larger significantly (maximum roughly 1.556 for temperature $1000^{\circ} \mathrm{C}$ and load velocity $2.0 \mathrm{E}-04 \mathrm{~mm} / \mathrm{s}$ ).

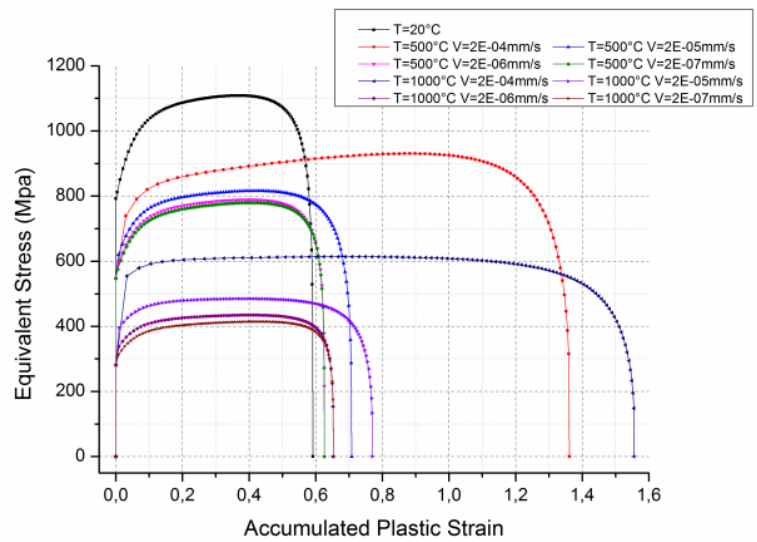

Figure 8. Stress-strain curves for different temperature and load velocity with micromorphic damage.

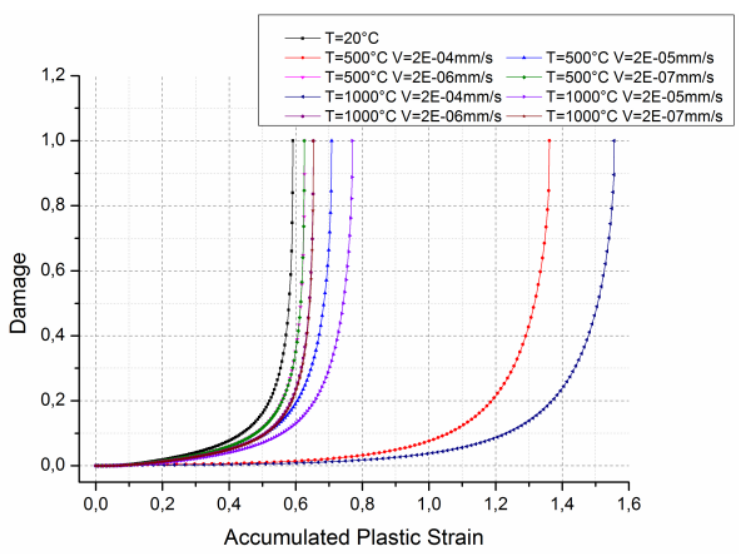

Figure 9. Evolution of damages for different temperature and load velocity with micromorphic damage.

\section{Conclusions}

The nonlocal anisothermal elasto-viscoplastic constitutive equations strongly coupled with ductile damage, nonlinear isotropic hardening and kinematic hardening 
are developed under the framework of the irreversible thermodynamic processes.

The new micromorphic damage is introduced into the principle of virtual power and a new balance equation is obtained. From the comparisons of the biaxial tension between local and micromorphic damage, we can make a conclusion that when micromorphic density and $\mathrm{H}$ modules approach to zero, the micromorphic effect is negligible and it degenerates to the local damage case; otherwise, the micromorphic damage significantly delays the evolution of local damage especially under cases of high temperature or high strain rate.

\section{Acknowledgement}

The financial support of ANR through the Program Micromorfing with contract ANR-14-CE07-0035-01 is fully acknowledged. The authors gratefully acknowledge financial support from China Scholarship Council.

\section{References}

1. K. Saanouni, Damage mechanics in metal forming: advanced modeling and numerical simulation. (John Wiley \& Sons, 2013).

2. E. Cosserat and F. Cosserat, Reprinted by Cornell University Library. English translation by $\mathrm{DH}$ Delphenich (2009).

3. R. D. Mindlin, Arch. Rational Mech. Anal. 16 (1), 51-78 (1964).

4. R. D. Mindlin, International Journal of Solids and Structures 1 (4), 417-438 (1965).

5. R. D. Mindlin and N. N. Eshel, International Journal of Solids and Structures 4 (1), 109-124 (1968).

6. A. C. Eringen and E. S. Suhubi, International Journal of Engineering Science 2 (2), 189-203 (1964).

7. A. C. Eringen, Microcontinuum field theories: I. Foundations and Solids. (Springer Verlag, New York, 1999).

8. S. Forest and R. Sievert, International Journal of Solids and Structures 43 (24), 7224-7245 (2006).

9. S. Forest, Journal of Engineering Mechanics 135 (3), 117-131 (2009).

10. C. Truesdell and W. Noll, The non-linear field theories of mechanics. (Springer, 2004).

11. K. Saanouni and M. Hamed, International Journal of Solids and Structures 50 (14-15), 2289-2309 (2013).

12. A. C. Eringen, Nonlocal continuum field theories. (Springer Science \& Business Media, 2002).

13. J. D. Lee and $X$. Wang, International Journal of Engineering Science 49 (12), 1378-1387 (2011).

14. S. Forest and E. C. Aifantis, International Journal of Solids and Structures 47 (25-26), 3367-3376 (2010).

15. D. P. Flanagan and T. Belytschko, International Journal for Numerical Methods in Engineering 17 (5), 679-706 (1981).

16. K. Saanouni, C. Forster and F. B. Hatira, International Journal of Damage Mechanics 3 (2), 140-169 (1994).

17. K. Saanouni and J. L. Chaboche, in Comprehensive Structural Integrity, edited by I. M. O. R. Karihaloo (Pergamon, Oxford, 2003), pp. 321-376.

18. H. Badreddine, K. Saanouni and A. Dogui, International Journal of Plasticity 26 (11), 1541-1575 (2010). 